\title{
Erwartungen aus deutscher verfassungsrechtlicher Sicht
}

\section{Einleitung}

Der Status quo der deutsche Gerichte ist gekennzeichnet durch eine Fremdverwaltung durch die Justizministerien der Länder bzw., soweit es um die Bundesgerichte geht, durch das Bundesjustizministerium. ${ }^{1}$ Zum Teil sind einige der fünf Fachgerichtsbarkeiten auch anderen Ressorts zugeordnet. Die Gerichtsverwaltung umfasst insbesondere die Personal-, Gebäude- und Finanzangelegenheiten. Teilzuständigkeiten in diesen Bereichen sind zwar von den Ministerien auf die Gerichtspräsidenten verlagert worden, doch handeln diese insoweit als Behördenleiter, die dem Ministerium und damit der exekutiven Gewalt untergeordnet sind. Lediglich in Personalangelegenheiten der Richter gibt es kleine Ansätze von Selbstverwaltung, da einige Bundesländer eine Mitwirkung von Vertretern der Justiz im Richterwahlausschuss vorsehen. Sie stellen aber in jedem Fall nur eine Minderheit der Mitglieder dieses Gremiums. Außerdem ist nach der herrschenden Auffassung zu jeder Entscheidung die Zustimmung des jeweiligen Justizministers erforderlich. ${ }^{2}$

Forderungen nach einer Änderung dieses im internationalen Vergleich relativ seltenen Zustands wurden in der Geschichte der Bundesrepublik mehrfach erhoben. Sie kommen jedoch meist nur aus den Richtervereinigungen, während sie in den politischen Parteien bisher wenig Unterstützung finden. ${ }^{3}$ Neuen Schwung hat die Diskussion in den letzten Jahren durch die Einführung von Elementen des Neuen Steuerungsmodells im Bereich der Justiz erhalten, da die Dezentralisierung der Ressourcenverantwortung ein zentrales Ziel dieses Managementkonzeptes ist, das die Frage der Eigenverantwortung der Justiz aufwirft. ${ }^{4}$ In der Rechtswissenschaft überwiegen bisher klar die ablehnenden Stimmen. Auf dem Juristentag 2002, der allerdings nicht repräsentativ zusammengesetzten Versammlung von Vertretern der verschiedenen juristischen Berufe, wurde die Forderung nach einer Selbstverwaltung der Justiz von der Mehrzahl der Redner abgelehnt. ${ }^{5}$

\section{Verfassungsrechtliche Rahmenbedingungen}

Weder das Grundgesetz noch die sechzehn Landesverfassungen enthalten eine ausdrückliche Regelung über das Verhältnis der Justiz zur Exekutive. Lediglich über die Wahl der Richter gibt es spezielle Regelungen in Art. 95 II, 98 IV GG und einigen

1 K.F. Röhl, JZ 2002, 838 (840); ausführlich Wittreck, Die Verwaltung der Dritten Gewalt, 2006, S. $266 \mathrm{ff}$.

2 Vgl. Schulze-Fielitz, in: Dreier (Hrsg.), GG, Bd. 3, 2. Aufl., 2008, Art. 98, Rn. 43 m.w.N.

3 Weber-Grellet, DRiZ 2006, 22 (25).

4 Dazu z.B. Eifert, DV 1997, 75 ff.; Groß, DV 2001, 371 (377 ff.); K.F. Röhl, JZ 2002, 838 ff.; Schütz, Der ökonomisierte Richter, 2005, S. 329 ff.

5 Vgl. die Beiträge in: Verhandlungen des 64. Deutschen Juristentages, Bd. II/1, 2002, S. Q 7 ff. 
Landesverfassungen, die zum Teil einen Richterwahlausschuss, in einigen wenigen Fällen auch die Wahl von Gerichtspräsidenten unmittelbar durch das Parlament vorsehen. Hier besteht eine große föderale Vielfalt. ${ }^{6}$ Die Auslegung dieser Regelungen ist jedoch oft strittig. Ihr Verständnis wird durch die allgemeine verfassungsrechtliche Diskussion beeinflusst, die Vorgaben aus grundlegenden Prinzipien des Grundgesetzes abzuleiten sucht. Die folgende Darstellung konzentriert sich deshalb auf diese grundlegenden Weichenstellungen.

Im Zentrum der deutschen Debatte steht dabei das Demokratieprinzip (1.). Daneben sind aber auch die Garantie der richterlichen Unabhängigkeit (2.) und das Gewaltenteilungsprinzip (3.) von Relevanz.

\section{Das Demokratieprinzip}

Da nach der deutschen Verfassungstradition im Bund wie in den Ländern nur das Parlament unmittelbar vom Volk gewählt wird, bedürfen die anderen Teile der Staatsgewalt, d.h. die Exekutive und die Judikative, einer mittelbaren demokratischen Legitimation. Für die Verwaltungsbehörden erfolgt sie in der Regel über die parlamentarische Einsetzung und Verantwortlichkeit der Regierung als Leitungsinstanz des gesamten administrativen Apparates. Seine hierarchische Struktur mit der Möglichkeit von Einzelweisungen wird als Instrument zur Sicherung der demokratischen Legitimation verstanden. Bei der rechtsprechenden Gewalt erfolgt die inhaltliche Legitimation dagegen allein durch das Gesetz, da die Unabhängigkeit der Richter eine Einflussnahme durch Weisungen kategorisch ausschließt. Daneben spielt für die Rechtsprechung nur die personelle Legitimation eine Rolle, also die Frage, durch wen die Richter ausgewählt werden.

Strittig ist, ob die für die Behörden geltende Regelform der Legitimation auch auf die Gerichtsverwaltung anzuwenden ist. Nach der herrschenden Auffassung handelt es sich hierbei um eine Form der Ausübung von Staatsgewalt, die grundsätzlich den gleichen Legitimationsstrukturen wie die Exekutive unterworfen sein muss. ${ }^{7}$ Deshalb wird die Zuständigkeit eines Ministers für die Justizverwaltung als normal angesehen, da er dem Parlament verantwortlich ist, während Formen der Selbstverwaltung als problematisch angesehen werden.

Als Alternative zur Zuständigkeit der Justizministerien ist sowohl eine unmittelbare Anbindung der Justiz an das Parlament als auch eine Selbstverwaltung durch von der Justiz selbst bestimmte Vertreter denkbar. Unstrittig ist die demokratische Legitimation bei einer direkten Zuständigkeit des Parlaments. Dieses Verfahren ist in manchen Bundesländern für die Wahl von Gerichtspräsidenten vorgesehen. Gleiches gilt, wenn

6 Vgl. die Überblicke bei Dietrich, Richterwahlausschüsse und demokratische Legitimation, 2007, S. 103 ff., und Wittreck (Fn. 1), S. 395 ff.; zur Diskussion nach dem 2. Weltkrieg vgl. Böttcher, in: Umbach/Clemens (Hrsg.), GG, Bd. 2, 2002, vor Art. 92 ff., Rn. 9 ff.

7 So etwa Böckenförde, Verfassungsfragen der Richterwahl, 1974, S. 72; Wittreck (Fn. 1), S. 118 ff., 660 ff.; Voßkuhle/Sydow, JZ 2002, 673 (677); Classen, Betrifft Justiz 2008, 333 (334 ff.). 
die Mitglieder von Richterwahlausschüssen oder von einem Justizverwaltungsgremium $^{8}$ durch das Parlament bestimmt werden.

Nicht als demokratisch werden dagegen Modelle angesehen, in denen Richterwahlausschüsse oder Justizverwaltungsgremien ganz oder überwiegend von den Richtern selbst gewählt werden, da dadurch keine Rückbindung an das Volk gewährleistet sei. Nach der herrschenden Auslegung des Demokratieprinzips ergibt sich aus ihm also ein absolutes Kooptationsverbot. ${ }^{9}$ Damit wird jedoch die Gestaltungsbefugnis des demokratischen Gesetzgebers zu stark eingeschränkt. Formen der Selbstverwaltung sind mit dem Gebot demokratischer Legitimation vereinbar, solange der parlamentarische Gesetzgeber die Auswahl nach Eignungskriterien sicherstellt und das Verfahren für die Wahl der Mitglieder eines Richterwahlausschusses oder eines Verwaltungsgremiums selbst festlegt. ${ }^{10}$ Außerdem bleiben diese Gremien der parlamentarischen Kontrolle unterworfen. Weil die Justiz keine frei-gestaltende, sondern eine gebunden-kontrollierende Aufgabe hat, kommt der personellen Legitimation funktionsbezogen eine ganz andere Rolle zu als bei der Exekutive, sie muss der Sicherung der Unabhängigkeit der Justiz dienen. Im Gegensatz zu den Beamten, die als verlängerter Arm des jeweiligen Ministers gelten, sind die Richter nur dem Gesetz unterworfen und können nur im Fall der Rechtsbeugung zur Rechenschaft gezogen werden. Allerdings wäre die Einführung eines nur von den Richtern legitimierten Gremiums eine deutliche Veränderung in der Gewaltenzuordnung, die deshalb einer Verfassungsänderung bedürfte. ${ }^{11}$

\section{Die organisatorische Dimension der richterlichen Unabhängigkeit}

Nach der überwiegenden Auffassung bezieht sich die Garantie der richterlichen Unabhängigkeit in Art. 97 GG allein auf die Ausübung der rechtsprechenden Gewalt, d.h. die spruchrichterliche Tätigkeit, während die Aufgaben der Gerichtsverwaltung davon nicht erfasst werden. ${ }^{12}$ Allerdings liegt es für eine sozialwissenschaftlich informierte Rechtsauslegung auf der Hand, dass nicht nur unmittelbar durch Sanktionen Einfluss auf die richterliche Tätigkeit genommen werden kann, sondern auch indirekt durch die Gestaltung der Arbeitsbedingungen, also durch Ressourcenzuweisungen, und natürlich auch durch Personalentscheidungen, durch die das Einsatzgebiet und das Gehalt eines Richters gestaltet werden.

In der Grundrechtsdogmatik hat sich die Erkenntnis, dass Rechte nicht nur durch unmittelbare Eingriffe gefährdet werden, in der Anerkennung einer verfahrens- und einer

8 Vgl. die Vorschläge der Neuen Richtervereinigung (http://www.nrv-net.de/main.php?id=15 1\&vo_id=292), des Bundesfachausschusses Richterinnen und Richter, Staatsanwältinnen und Staatsanwälte in der Gewerkschaft ver.di (Verdikt Nr. 2/2003, S. 19 ff., abrufbar unter www.verdikt.verdi.de) und des Deutschen Richterbundes (http://www.drb.de/cms/index. php?id=111).

9 So z.B. Dreier, in: ders. (Hrsg.), GG, Bd. 2, 2. Aufl., 2006, Art. 20 (Demokratie), Rn. 143; Wittreck (Fn. 1), S. 131 f. m.w.N.

10 Groß, ZRP 1999, 361 (364); ebenso, aber für eine Wahl der richterlichen Mitglieder durch das Parlament Tschentscher, Demokratische Legitimation der Dritten Gewalt, 2006, S. $254 \mathrm{ff}$.

11 Groß, ZRP 1999, 361 (364).

12 Böckenförde (Fn. 7), S. 68 f.; Papier, NJW 2002, 2585 (2587 f.). 
organisationsrechtlichen Schutzdimension der Grundrechte niedergeschlagen. ${ }^{13}$ Die gleichen Überlegungen sind aber auch auf die richterliche Unabhängigkeit übertragbar, die ebenfalls durch eine flankierende organisatorische Komponente ergänzt werden muss. ${ }^{14}$ Auch wenn ein empirischer Nachweis naturgemäß kaum möglich ist, gibt es hinreichend viele Hinweise, dass der Einfluss der Justizministerien auf die Karriere von Richtern Rückwirkungen auf ihr Entscheidungsverhalten hat, auch wenn die richterliche Unabhängigkeit formal nicht angetastet wird. ${ }^{15}$ Warum haben so viele andere Staaten besondere organisatorische Schutzmechanismen geschaffen, wenn es gar keinen Grund für Misstrauen gegenüber der Exekutive gäbe?

\section{Die Gewaltenteilung}

Das in Art. 20 Abs. 2 S. 2 GG enthaltene Gewaltenteilungsprinzip, wonach die Staatsgewalt durch besondere Organe der Gesetzgebung, der vollziehenden Gewalt und der Rechtsprechung ausgeübt wird, verlangt keine strikte organisatorische Trennung der drei verschiedenen Funktionen. Vielmehr ergeben sich aus dem parlamentarischen Regierungssystem und anderen Regelungen der Verfassung Verschränkungen der Gewalten, so dass heute vielmehr von einer Funktionenordnung oder Gewaltengliederung gesprochen wird. $^{16}$ Dennoch setzt das Gewaltenteilungsprinzip die grundsätzliche »Besonderung« der Organe voraus, da andernfalls die konstitutive Kontrollfunktion der Gewalten untereinander nicht sinnvoll ausgeübt werden kann.

Gerade im Verhältnis der Judikative zur Exekutive ist diese Kontrollfunktion offensichtlich. Sie umfasst neben den drei öffentlich-rechtlichen Gerichtszweigen der Verwaltungs-, Sozial- und Finanzgerichtsbarkeit außerdem die ordentliche Gerichtsbarkeit, da die Zivilgerichte auch über Staatshaftungsansprüche und die Strafgerichte über Straftaten von Amtsträgern entscheiden, und ebenso die Arbeitsgerichtsbarkeit, die für arbeitsvertragliche Streitigkeiten im öffentlichen Dienst zuständig ist. ${ }^{17}$ Folglich können Möglichkeiten der Einflussnahme der Exekutive auf die Gerichte, insbesondere im heiklen Bereich der sog. Beförderung von Richtern auf höher bewertete Posten, eine Gefährdung ihrer Unabhängigkeit und damit eine Schwächung der Kontrolle bedeuten. Insbesondere bei den öffentlich-rechtlichen Gerichtsbarkeiten spricht deshalb das Gewaltenteilungsprinzip deutlich für eine strikte organisatorische Trennung von der Exekutive auch im Bereich der Justizverwaltung. ${ }^{18}$

Von den Verteidigern des Status quo wird das Gewaltenteilungsprinzip jedoch umgekehrt eingesetzt. Sie argumentieren, dass insbesondere die Verwaltung des Justiz-

13 Vgl. z.B. Schmidt-Aßmann, in: Merten/Papier (Hrsg.), Handbuch der Grundrechte, Bd. 2, $2006, \S 45$.

14 Dazu näher Groß, DV Beiheft 5, 2002, 217 (218 ff.); ähnlich Berlit, DV Beiheft 5, 2002, 135 (149 ff.); anders Schuppert, in: Hoffmann-Riem (Hrsg.), Reform der Justizverwaltung, 1998, S. 215 (235).

15 Schulte-Kellinghaus, ZRP 2008, 205 (207).

16 Vgl. Poscher, in: Hoffmann-Riem/Schmidt-Aßmann/Voßkuhle (Hrsg.), Grundlagen des Verwaltungsrechts, Bd. 1, 2006, § 8; Möllers, Gewaltengliederung, 2005, passim.

17 Groß, ZRP 1999, 361 (361).

18 So auch Weber-Grellet, DRiZ 2006, 22 (25). 
personals eine originär exekutive Aufgabe sei. ${ }^{19}$ Dieser Hinweis greift jedoch offensichtlich zu kurz, denn dann müsste auch das Personal der Parlamente, der Rechnungshöfe oder der Verfassungsgerichte von einem Regierungsmitglied oder zumindest einer weisungsunterworfenen Stelle ausgesucht werden. Das wird aber ersichtlich von niemandem postuliert. Vielmehr zeigt sich hier, dass Ressourcenentscheidungen im Regelfall von organisatorisch selbständigen Einheiten auch selbst verantwortet werden. Prinzipienwidrig ist die Fremdverwaltung der Justiz, nicht ihre Selbstverwaltung.

\section{Rechtspolitische Hintergründe}

Dass die wissenschaftliche Diskussion bei der Auslegung von grundlegenden Bestimmungen der Verfassung zu teilweise diametral entgegengesetzten Ergebnissen führt, ist nicht besonders ungewöhnlich. Dennoch lohnt sich auch angesichts der manchmal polemischen Elemente in der Auseinandersetzung ein kurzer abschließender Blick auf mögliche Erklärungen für die jeweiligen Positionen. Ich sehe hier drei Ansatzpunkte. Zum einen spürt man eine gewisse Furcht vor der Macht der Richter (1.). Zum zweiten gibt es manchmal ein erstaunliches Vertrauen in die Exekutive (2.). Schließlich ist drittens eine gewisse nationale Introvertiertheit der deutschen Diskussion immer noch zu beobachten (3.).

\section{Furcht vor der Macht der Richter?}

Ein häufiges Argument gegen eine weitgehende Selbstverwaltung der Justiz ist die Furcht vor der Macht der Richter. Dabei wird im Ansatz sicher zutreffend auf die praktischen Grenzen der Bindung an das Gesetz hingewiesen. ${ }^{20}$ Richter sind keine Rechtsprechungsautomaten, die vom Gesetzgeber programmiert werden. In der justizstaatlichen Tradition Deutschlands mit einer relativ engmaschigen gerichtlichen Kontrolltätigkeit gerade gegenüber der Exekutive ${ }^{21}$, noch dazu mit einer starken Verfassungsgerichtsbarkeit, die auch dem Gesetzgeber Grenzen setzt, erscheinen die Richter so als eine besonders einflussreiche Gruppe, deren Tätigkeit auch eine hohe politische Relevanz hat. Daraus ergibt sich dann offensichtlich die Folgerung, dass die politisch Verantwortlichen in der Regierung, wenn sie schon keinen direkten Einfluss auf die rechtsprechende Tätigkeit selbst ausüben können, wenigstens deren Rahmenbedingungen, und hier insbesondere die Personalentscheidungen kontrollieren müssen, um das Entstehen eines »Staates im Staate $«$ zu verhindern. ${ }^{22}$ Immer wieder findet sich deshalb auch die Beschwörung einer Verselbständigung des »Richterstandes «, falls eine Selbstverwaltung eingeführt würde. ${ }^{23}$

19 Ehlers, Verfassungsrechtliche Fragen der Richterwahl, 1998, S. 35 ff.; Mertin, ZRP 2002, 332 (334); Dästner, DV Beiheft 5, 2002, S. 201 (213 f.).

20 Röhl, JZ 2002, 838 (846).

21 Vgl. Groß, ZÖR 2006, 625 ff.

22 Vgl. Hoffmann-Riem, DRiZ 2003, 284 (291): »Justiz als isolierte Gewalt?«.

23 Z.B. Böckenförde (Fn. 7), S. 109; Wassermann, AK-GG, 3. Aufl., 2001, Art. 92, Rn. 13a; Wittreck (Fn. 1), S. 653 f.; krit. Schulte-Kellinghaus, ZRP 2008, 205 (207 f.). 
Dahinter steht wohl auch die historisch durchaus reale Erfahrung einer überwiegenden Republikfeindlichkeit der Richter in der Weimarer Zeit, als die Dritte Gewalt in vielen Fällen tatsächlich keine Stütze der geltenden Verfassung war. ${ }^{24} \mathrm{Ob}$ eine solche Entwicklung allerdings nach sechzig Jahren konsolidierter Demokratie und einer auch sozial deutlich gemischteren Besetzung der Richterschaft denkbar wäre, ist zu bezweifeln. Ein valides Argument gegen eine vernünftig organisierte Eigenverantwortung der Judikative lässt sich aus der Geschichte nicht ableiten.

\section{Besonderes Vertrauen in die Exekutive?}

In Teilen der deutschen Rechtswissenschaft ist eine eigenartige Tradition zu beobachten, nämlich ein besonderes Vertrauen in die Gemeinwohlorientierung der Exekutive. In einer auf die monarchischen Verfassungen des 19. Jahrhunderts zurückgehenden Argumentationslinie wird insbesondere der Ministerialbürokratie eine zentrale Funktion bei der Leitung des Gemeinwesens auch im Verhältnis gegenüber Parlament und Gerichten zugewiesen. ${ }^{25}$ Die erwähnte Stärkung der gerichtlichen Kontrolle nach dem 2. Weltkrieg steht dazu nicht im Widerspruch, sondern kann vielmehr als komplementäre Gegenreaktion aufgefasst werden.

In der justizbezogenen Debatte äußert sich diese Rollenzuweisung an die Exekutive in verschiedenen Argumenten. Zum einen wird die Justizverwaltung als »Balancierungs- und Neutralisierungselement « gegenüber einer möglichen Parteipolitisierung der Richterschaft angesehen. ${ }^{26}$ Das ist blauäugig gegenüber der Realität der ministeriellen Personalpolitik. Zum zweiten wird behauptet, dass die Regierung eine besondere Verantwortung für die Verwirklichung des Justizgewährleistungsanspruchs trage und deshalb die Kontrolle über die Ressourcen der Gerichte behalten müsse. ${ }^{27}$ Die Gesamtverantwortung für die Funktionsfähigkeit der Justiz und ihre adäquate Ressourcenausstattung liegt aber zweifelsohne beim Parlament als Organisations- und Haushaltsgesetzgeber. Auch wird drittens der Dienstaufsicht durch die Exekutive eine wichtige Funktion bei der Sicherung der Rechtsbindung zugewiesen. ${ }^{28}$ Warum sollte es aber nicht möglich sein, justizinterne Mechanismen der Qualitätssicherung zu entwickeln $?^{29}$

\section{Nationale Introvertiertheit?}

Zwar kommt in neuerer Zeit kaum ein Diskussionsbeitrag zur Stellung der Judikative ohne eine Stellungnahme zur Sonderstellung der deutschen Justiz in der Europäischen Union aus. ${ }^{30}$ Zum Teil wird jedoch die Rechtsentwicklung in den anderen europä-

24 Zusammenfassend Gusy, Die Weimarer Reichsverfassung, 1997, S. 221 ff.

25 Günther, Denken vom Staat her, 2004, S. 29 ff., 198 f. u.ö.

26 Böckenförde (Fn. 7), S. 107.

27 So wohl Hoffmann-Riem, DV 1997, 481 (493).

28 Wittreck (Fn. 1), S. 142 ff.

29 Überlegungen dazu bei Berlit, DV Beiheft 5, 2002, 135 (168 ff.).

30 Vgl. etwa die Darstellungen bei Tschentscher (Fn. 10), S. 270 ff., und Wittreck (Fn. 1), S. $527 \mathrm{ff}$. 
ischen Ländern mit dem Hinweis abgetan, dass ein umfassender Systemvergleich der Staatsverfassungen notwendig sei. ${ }^{31}$ Mit diesem Argument kann man letztlich jede rechtspolitische Funktion eines Verfassungsvergleichs aushebeln, denn natürlich gleichen sich Verfassungen kaum in allen wesentlichen Grundstrukturen, und diese sind gerade in Fragen der Gewaltenteilung betroffen.

Außerdem wird auf Schwächen der Gerichtsbarkeit in anderen Ländern hingewiesen, wobei insbesondere Italien ein dankbares Beispiel ist. ${ }^{32}$ Nach wie vor ist eine hohe Zahl von Verurteilungen durch den Europäischen Gerichtshof für Menschenrechte wegen überlanger Verfahrensdauer zu beklagen. Damit ist allerdings nicht gesagt, dass die Selbstverwaltungsstrukturen dafür ursächlich sind. Aus anderen europäischen Ländern mit einer größeren Selbständigkeit der Justiz sind ähnliche Probleme insbesondere der Effizienz nicht bekannt. Ein wesentlicher Faktor ist vielmehr das Prozessrecht. Andererseits zeigen gerade die Manöver der verschiedenen von Berlusconi geführten Regierungen, wie wichtig eine weitgehende Unabhängigkeit der Justiz zur Gewährleistung der demokratischen Verfassungsordnung sein kann. Deshalb kommt es vielmehr darauf an, sich genauer mit den Stärken und Schwächen der ausländischen Modelle auseinander zu setzen. Hierzu leistet diese Tagung einen begrüBenswerten Beitrag. 